\title{
Implementation of the Adaptive CSMA-CA Algorithm Based on Traffic Load
}

\author{
Lin Zhu ${ }^{1}$, Guangming $\mathrm{Li}^{1 *}$ and Wei Liu ${ }^{1}$ \\ ${ }^{1}$ School of Mechanical, Electrical and Information Engineering, \\ Shandong University, Weihai, 264209, China \\ zhulingshandong@gmail.com,gmli@sdu.edu.cn,liuwei1206.sw@163.com
}

\begin{abstract}
IEEE802.15.4 is a standard designed for low rate wireless personal area network. For its MAC protocol adopts the competition method to share wireless channel, collisions between ending devices are unavoidable and lead to retransmissions, consuming more energy. As traffic load becomes heavier, collisions and retransmissions are more serious. The focus of this paper is to achieve dynamic-parameter CSMA-CA algorithm based traffic load in IEEE 802.15.4 and measure the performance of modified IEEE802.15.4 in some aspects (packet delivery rate, collision rate, collision rate and average MAC delay). Different from previous papers, it analyzes the performance of the algorithm in direct data transmission and respectively executes simulations both including and excluding hidden-terminal (HT) problems.
\end{abstract}

Keywords: IEEE 802.15.4; CSMA-CA; dynamic-parameter; direct data transmission

\section{Introduction}

In recent years, the concept of wireless sensor networks (WSNs) has gradually penetrated into various applications such as intelligent transportation, environment monitoring, and smart home for its features of low power consumption, low cost, short transmission distance, moderate response time and good survivability.

Serving as one of promising candidates for WSNs, IEEE 802.15.4 is a short-distance wireless communication standard. Low power consumption is the most important feature of the standard. Previous work for IEEE802.15.4 can be classified roughly into the following cases. Various performance metrics and experimental scenes are designed to evaluate the performance of IEEE 802.15.4 [1]. Markov chains based analytical model are proposed to evaluate the slotted CSMA-CA algorithm in the MAC layer. MAC protocol with ACK frame transmission [2], retransmissions [3] and throughput limit [4] are studied with the above-mentioned models. Bottlenecks of IEEE 802.15.4 are analyzed and different mechanisms are proposed to enhance its performance [5]. A two-stage adaptive contention control strategy is implemented and evaluated on hardware to improve transmission efficiency in indirect transmission type [6], but the commonly direct data transmission and hidden-terminal problems are not considered. In this paper, the influence of traffic load on performance metrics of IEEE802.15.4 is study. The memorized adaptive scheme is extended to direct data transmission type and the performance of the adaptive algorithm is examined in two situations, considering or removing hidden-terminals.

The rest of this paper is organized as follows: In Section 2, we give a brief description of IEEE 802.15.4 MAC layer. Next, in Section 3, the adaptive CSMA-CA algorithm is introduced. In Section 4, the analysis model of the modified is given. Then we present the 
experimental setup and give out the experimental results with discussions in Section 5. Finally, in Section 6, we present the conclusion.

\section{A Brief Description of IEEE 802.15.4 MAC Layer}

According to IEEE 802.15.4 specification [7], the MAC sublayer provides an interface between the service specific convergence sublayer (SSCS) and the PHY layer. Its main functions include the following aspects. The coordinator generates beacons periodically and devices synchronize with the coordinator by tracking beacons. Association and disassociation are applied to support self-configuration. Devices contend channel by the carrier sense multiple access with collision avoidance mechanism (CSMA-CA). The reliability of the link between two peers is enhanced by various mechanisms, such as frame acknowledgment, retransmission and data verification. In this subsection, we give a description of items closely related to our performance study, including beacon-enabled mode, data transfer types.

\subsection{Bacon-enabled Mode}

An IEEE 802.15.4 network can operate in either beacon-enabled mode or non beacon-enabled mode. In non beacon-enabled mode devices can send data at any time based on CSMA/CA. While in beacon-enabled mode, devices must synchronize with the coordinator and send data based on CSMA/CA at the boundaries of time units called backoff slots.

In IEEE 802.15.4 standard, a superframe structure is used in beacon-enabled mode to manage periodic beacon transmissions and data transmissions. The superframe is bounded by network beacons sent by the coordinator. It includes three parts: a beacon transmission part, an active part and an inactive part. At the beginning of a superframe, a beacon shall be transmitted. Then the active part shall commence. According to different transmission modes, the active part is divided into a contention access period (CAP) and a contention-free period. During the CAP, any device wishing to communicate competes with other devices using the slotted CSMA-CA mechanism. The CFP, if present, follows immediately after the CAP and extends to the end of the active part. Data transmissions within the CFP shall be completed in certain slots allocated by the coordinator using guaranteed time slots (GTSs) mechanism. All transmissions must be suspended when the inactive part arrives and devices in PAN enter a low-power mode for reducing power consumption.

\subsection{Data Transfer Types}

Direct data transmission applies to all data transmissions either from a device to a coordinator, from a coordinator to a device, or between two peers. In this transfer type, data frames contend channel by the CSMA-CA algorithm.

Indirect data transmission only applies to data transmissions from a coordinator to devices. In this transfer type, all data frames sent by the coordinator will be kept in the transaction list and the coordinator notifies corresponding devices to extract pending data by beacon frame.

GTS data transmission applies to data transmission between a device and its coordinator regardless of transmission directions. It's designed for low-latency applications or applications requiring specific data bandwidth. In this data transfer type, the coordinator may dedicate portions of the active superframe to some special applications.

An adaptive contention control strategy based on a two-stage approach has been introduced to improve performance in indirect transfer [6]. In this paper, a various-parameter CSMA-CA algorithm based traffic load is achieved in direct data transfer and a performance analysis is given in details. 


\section{Adaptive Contention Strategy}

\subsection{IEEE 802.15.4 CSMA-CA Mechanism}

In the beacon-enabled mode, the slotted CSMA/CA is used when devices access channel. Three counters, BE, CW and NB are maintained for channel access control. BE defines backoff exponent used by devices to draw the random backoff value. $\mathrm{CW}$ defines the number of backoff slots that the channel needs to be sensed idle continuously before a transmission. NB defines the number of backoffs that the CSMA-CA algorithm has attempted before accessing to the channel.

Then the slotted-CSMA algorithm works as follows. All associated devices synchronize with the pan coordinator. Before a new transmission, BE, CW and NB are initialized to macMinBE, 2 and 0. Devices with data frames to send first backs off for a random number of slots within $\left(2^{\mathrm{BE}}-1\right)$. Then after the random time, the device executes CCA, which is short for clear channel assessment. If two CCAs notify that the channel is idle, the device will send the data frame. If either of CCAS notifies that the channel is busy, BE and NB are increased by one and $\mathrm{CW}$ is reset to 2.The above procedure is repeated until the device transmits the frame or NB arrives at macMaxCSMABackoffs.

\subsection{Motivation}

Since IEEE 802.15.4 is originally designed for low-rate wireless personal area network, the system behaves well on low traffic load and suffers a serious decline on heavy traffic load. Based on the CSMA-CA algorithm described in the previous section, an amount of contention overheads result from iterative backoff operations when the system traffic load is heavy. It can be illustrated as follow. The backoff window will be reset to the minimum value before a new transmission. When network load is heavy, the serious contention could not be resolved within a narrow backoff window, which leads to more collisions.

To improve system performance under various network loads, an adaptive CSMA-CA algorithm is needed. It is applicable for wireless networks in which traffic loads do not change fast.

Through previous experiments and theoretical analysis, the values of MAC parameters have an impact on the performance of the network [2]. When frames arrive at a moderate rate, devices with a smaller backoff window transmit the frames more quickly and have a higher throughput. On the other hand, when frames arrive at a higher rate, devices with a larger backoff window behave better for reducing collisions and improving transmission efficiency. Therefore, we make the parameter, initial back off window, changes with traffic loads for a better performance.

\subsection{Adaptive CSMA-CA Algorithm Flow}

According to the standard CSMA-CA algorithm, the mean of parameter BE in successful data deliveries in a certain interval can reflect current traffic load. Devices have a higher probability to access the channel with a small backoff window when network load is light. As traffic load becomes heavier, devices have a higher probability to try more times for channel access which leads a large backoff window. So we choose the mean of BE in successful transmissions during a period to predict the initial backoff window in the next period.

So the adaptive algorithm is implemented in the following flow, as shown in Figure 1. The $\mathrm{BE}$ value for the successful data delivery in the current superframe is recorded to predict the initial value of the backoff window for the next superframe. Then at the beginning of the next superframe, the pan coordinator announces the initial backoff exponent value which is 
defined as adaptiveBE, to end devices via the beacon frame. When the next superframe starts, devices shall employ this suitable BE matching the current traffic load in the CSMA-CA. In addition, when traffic load becomes lighter, the adaptiveBE should be decreased gently to avoid a too widen backoff window.

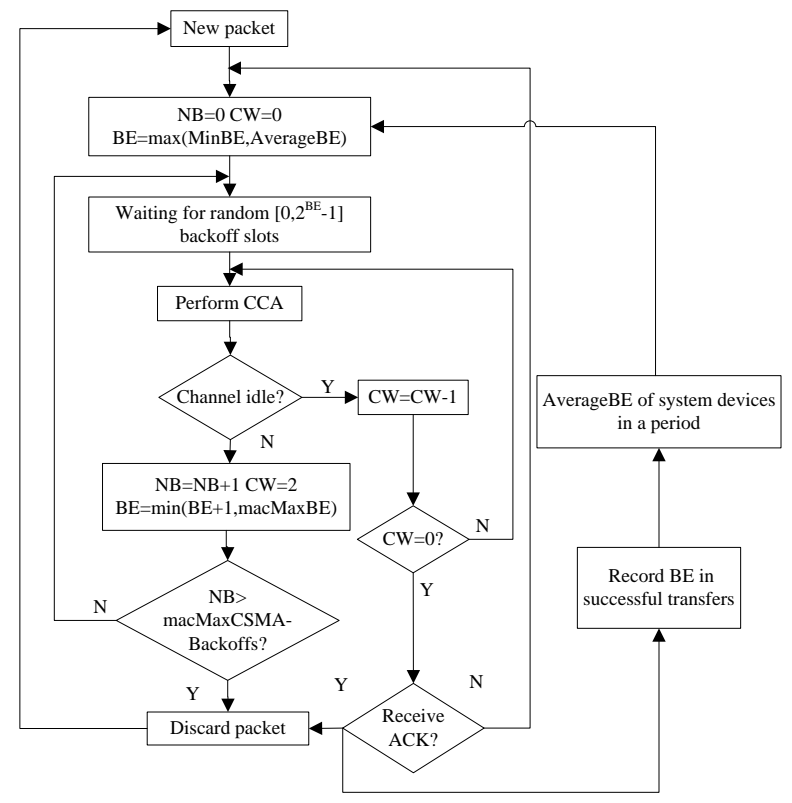

Figure 1. Adaptive CSMA-CA Algorithm Flowchart

\section{Performance Analysis}

We now introduce analytical model. Some notations used in this part are listed in Table 1.

Table 1. Notations in the Performance Analysis

\begin{tabular}{|l|l|}
\hline$N$ & Number of ending devices. \\
\hline$L_{\text {data }}$ & Data frame length. \\
\hline$L_{\text {ack }}$ & ACK frame length. \\
$L_{\text {duration }}$ & $\begin{array}{l}\text { The waiting duration between data frame and } \\
\text { ACK frame. }\end{array}$ \\
\hline$\lambda$ & Packet arrival rate of an ending device. \\
\hline$G$ & System output. \\
\hline$m$ & $\begin{array}{l}\text { The maximum number of backoff operations } \\
\text { before declaring a channel access failure. }\end{array}$ \\
\hline$n$ & $\begin{array}{l}\text { The maximum number of retries allowed after } \\
\text { a transmission failure. }\end{array}$ \\
\hline
\end{tabular}

In the analysis, we assume a beacon-enabled star topology wireless network with ${ }_{N}$ ending devices. The data frame length is assumed $L_{\text {data }}$ back-off slots and the arrival process of frames follows a Poisson distribution with a certain arrival rate $\lambda$. In the following part, we analyze $G$ for the adaptive CSMA-CA. Here, we focus on the unsaturated case in ACK mode. 
Given the aforementioned assumption, the behavior of the system can be analyzed by Markov chain models of a single device and the channel. In the following part, the behavior of a single device is analyzed using a 3-D Markov chain. Each state is indicated by $\{r(t)=i, s(t)=j, b(t)=k\},\left\{b_{T_{i}}\right\}$ or $\left\{b_{A C K_{i}}\right\}$. At a given backoff slot $\mathrm{t}$, the stochastic process $r(t)$ represents the times of retransmission for not receiving ACK frame, $s(t)$ represents the transmission stage and $b(t)$ represents the backoff stage. Then the steady state probability of being in each state is listed as follow

$$
\begin{aligned}
& \left\{b_{i, j, k}=\lim _{t \rightarrow \infty} P\{r(t)=i, s(t)=j, b(t)=k\}\right. \\
& \left\{b_{T_{i}}=\lim _{t \rightarrow \infty} P\left\{T X_{r(t)}=T X_{i}\right\}\right. \\
& b_{A C K_{i}}=\lim _{t \rightarrow \infty} P\left\{A C K_{r(t)}=A C K_{i}\right\} \\
& b_{\text {idle }}=\lim _{t \rightarrow \infty} P\{r(t)=-1, s(t)=-1, b(t)=-1\}
\end{aligned}
$$

In this model, $p_{i}^{c}$ is the probability that the channel is idle and $p_{\left.i\right|_{i} ^{c}}^{c}$ is the probability that the channel is idle in next backoff slot given that channel is idle in the current backoff slot. By inspecting Figure 2, each steady-state probability can be related by the following Eqs. (2)-(6).

$$
\begin{aligned}
& b_{\text {idle }}=(1-p) b_{\text {idle }}+\left(1-p_{i}^{c}\right) \sum_{i=1}^{n} b_{i, m, 0}+\left(1-p_{i \mid i}^{c}\right) \sum_{i=1}^{n} b_{i, m,-1}+q \sum_{i=1}^{n-1} b_{A C K_{i}}+b_{A C K_{n}} \\
& b_{i, j, 0}=\left\{\begin{array}{lr}
(1-q) b_{A C K_{i-1}} / W_{1}+b_{i, j, 1}, & 2 \leq i \leq n, j=1 \\
{\left[\left(1-p_{i}^{c}\right) b_{i, j-1,0}+\left(1-p_{i \mid i}^{c}\right) b_{i, j-1,-1}\right] / W_{j}+b_{i, j, 1}, 1 \leq i \leq n, 2 \leq j \leq m} \\
p b_{\text {idle }} / W_{1}+b_{1,1,1}, & i=1, j=1
\end{array}\right. \\
& b_{i, j, k}=\left\{\begin{array}{lc}
(1-q) b_{A C K_{i-1}} / W_{1}+b_{i, j, k+1}, & 2 \leq i \leq n, j=1 \\
{\left[\left(1-p_{i}^{c}\right) b_{i, j-1,0}+\left(1-p_{i \mid i}^{c}\right) b_{i, j-1,-1}\right] / W_{j}+b_{i, j, k+1},} & 1 \leq i \leq n, 2 \leq j \leq m \\
p b_{\text {idle }} / W_{1}+b_{1,1, k+1}, & i=1, j=1
\end{array}\right. \\
& b_{i, j,-1}=p_{i}^{c} b_{i, j, 0} \\
& b_{A C K_{i}}=b_{T_{i}}=p_{i \mid i}^{c} \sum_{j=1}^{m} b_{i, j,-1}
\end{aligned}
$$

In Eq. (4), we give the expression of steady state $b_{i, j, k}$ when $1 \leq k<W_{i}-1$. The expression form of $b_{i, j, k}$ when $k=W_{i}-1$ only include the first part of Eq. (4). 


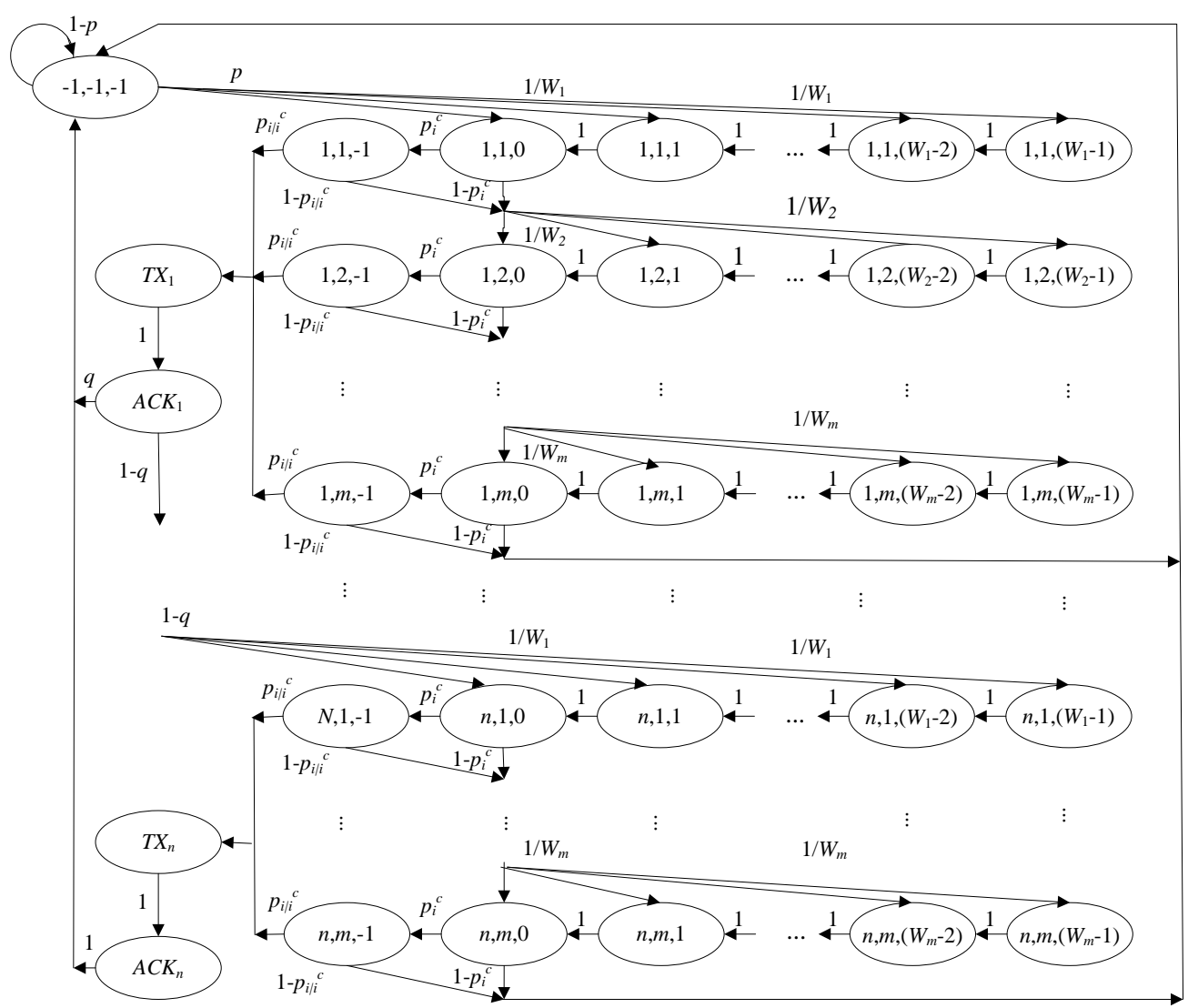

Figure 2. Markov Model of One Single Node for Slotted CSMA/CA

We use the above assumption to calculate some metrics which determine the device model. According to the adaptive algorithm, the initial backoff window is different from standard IEEE 802.15.4. It is the same as the standard at the beginning but changes with system traffic load in the following periods, and then we calculate the backoff window in next period based on traffic load in the current period by

$$
\begin{gathered}
W_{1}=q p_{i}^{c} p_{i \mid i}^{c} \sum_{i=1}^{n} \sum_{j=1}^{m} \sum_{k=-1}^{w_{y}-1} b_{i, j, k} \times k \\
W_{j}= \begin{cases}2^{j-1} W_{1}, j \leq \log _{2}\left(2^{m+1} / W_{1}\right) \\
2^{m}, \quad \log _{2}\left(2^{m+1} / W_{1}\right)<j \leq m\end{cases}
\end{gathered}
$$

The probability of a frame arriving at the device in a certain back off slot is $p=\lambda / L_{\mathrm{data}}$. The probability of a frame being sent has a collision with other frames can be derived as $q=\left(1-p_{t \mid i i}^{c}\right)^{N-1}$ and the probability $p_{t \mid i}$ of a device beginning a transmission given that the channel is idle for two backoff slots is equal to

$$
p_{t \mid i i}=p_{t} / p_{i i}^{c}=\sum_{i=1}^{n} \sum_{j=1}^{m} b_{i, j,-1} / p_{i}^{c}\left(b_{\text {idle }}+\sum_{i=1}^{n} \sum_{j=1}^{m} \sum_{k=-1}^{w_{m}-1} b_{i, j, k}+L_{\text {data }} \sum_{i=1}^{n} b_{T_{i}}+\left(L_{\text {duration }}+L_{\text {ack }}\right) \sum_{i=1}^{n} b_{A C K_{i}}\right)
$$


For it's easy to get $p_{i \mid b}^{c}$ which is the probability that the channel become idle in the next backoff slot given that it's busy in the current back off slot, $p_{\left.i\right|_{i} ^{c}}^{c}$ can be simplified as the following form.

$$
\begin{gathered}
p_{i \mid b}^{c}=2 /\left(L_{\mathrm{data}}+L_{\mathrm{ack}}\right) \\
p_{i \mid i}^{c}=\left[p_{i}^{c}-p_{i \mid b}^{c}\left(1-p_{i}^{c}\right)\right] / p_{i}^{c}=1-2\left(1-p_{i}^{c}\right) / p_{i}^{c}\left(L_{\mathrm{data}}+L_{\mathrm{ack}}\right)
\end{gathered}
$$

To numerically obtain $p_{i}^{c}$, we take into account the joint distribution of the $N$ devices which decides the channel state. In the previous work [2], the channel modeled by a discrete-time Markov chain in Fig. 3 has been proposed and we use it for our analysis.

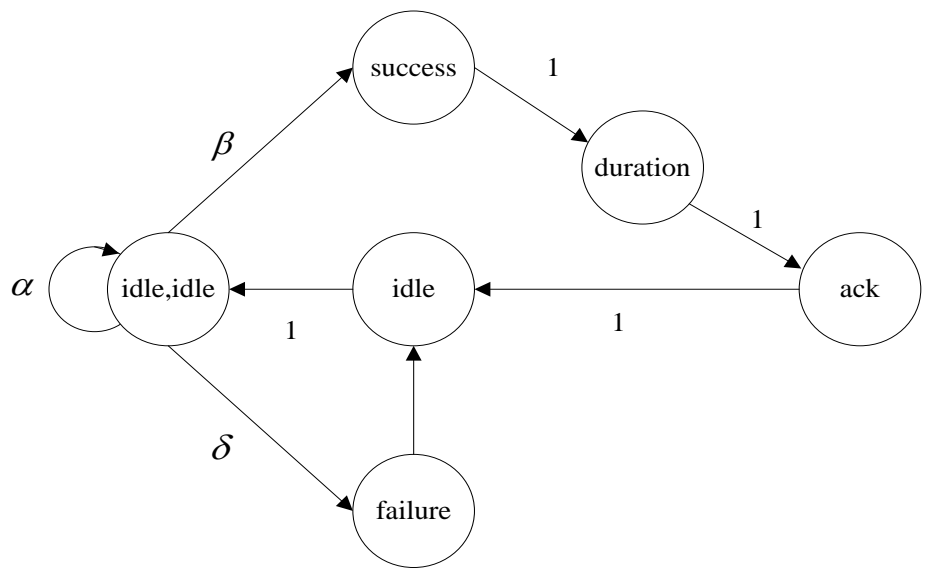

Figure 3. Markov Model of the Channel

Let $b_{\text {idle, idle }}^{c}$ be the steady probability that the channel is idle. The channel will be remaining in this state with the probability $\alpha$ if no devices begin a transmission at the next backoff slot. If only one device begins transmission, the channel will move to $b_{\text {success }}^{c}$ with the probability $\beta$. If more than one device begins transmission at the same time, the channel will move to $b_{\text {failure }}^{c}$ with the probability $\delta$. Then $\alpha, \beta, \delta$ are calculated by Eqs. (12-14), and each steady state of the channel can be expressed by $\alpha, \beta$.

$$
\begin{gathered}
\alpha=\left(1-p_{t \mid i i}\right)^{N} \\
\beta=N p_{t \mid i i}\left(1-p_{t \mid i i}\right)^{N-1} \\
\delta=1-\left(1-p_{t \mid i i}\right)^{N}-N p_{t \mid i i}\left(1-p_{t \mid i i}\right)^{N-1}
\end{gathered}
$$

The probability $p_{i i}^{c}$ of the channel remains idle for two consecutive backoff slots is derived as the Eq. (15).

$$
\begin{aligned}
p_{i i}^{c} & =b_{\text {idle, idle }}^{c} /\left(b_{\text {idle, idle }}^{c}+L_{\text {data }} b_{\text {success }}^{c}+L_{\text {duration }} b_{\text {duration }}^{c}+L_{\text {ack }} b_{\text {ack }}^{c}+L_{\text {data }} b_{\text {failure }}^{c}+b_{\text {idle }}^{c}\right) \\
& =1 /\left[\left(L_{\text {data }}+1\right)(1-\alpha)+\left(L_{\text {ack }}+L_{\text {duration }}\right) \beta+1\right] \\
p_{i}^{c}=p_{i i}^{c} / p_{i \mid i}^{c} & =\left\{\left(L_{\text {data }}+L_{\text {ack }}\right) /\left[\left(L_{\mathrm{data}}+1\right)(1-\alpha)+\left(L_{\text {ack }}+L_{\text {duration }}\right) \beta+1\right]\right\} \times 1 /\left(L_{\text {data }}+L_{\text {ack }}+2\right)
\end{aligned}
$$


According to Eqs. (11), (15), and (16), the constraints in the following Eqs. (17, 18), the value of $p_{i}^{c}$ can be calculated.

$$
\begin{gathered}
b_{\text {idle, idle }}^{c}+L_{\text {data }} b_{\text {success }}^{c}+L_{\text {duration }} b_{\text {duration }}^{c}+L_{\text {ack }} b_{\text {ack }}^{c}+L_{\text {data }} b_{\text {failure }}^{c}+b_{\text {idle }}^{c}=1 \\
b_{\text {idle }}+\sum_{i=1}^{n} \sum_{j=1}^{m} \sum_{k=-1}^{w_{y}-1} b_{i, j, k}+L_{\text {data }} \sum_{i=1}^{n} b_{T_{i}}+L_{\text {ack }} \sum_{i=1}^{n} b_{A C K_{i}}=1
\end{gathered}
$$

As a result, metrics $q, p_{i \mid i}^{c}, \alpha, \beta$ and each state of two models are all available numerically. Base on the analysis above, the system throughput which is defined as the ratio of data transmission periods to all time that a transmission procedure spends can be expressed as the following equation.

$$
G=L_{\text {data }} b_{\text {success }}^{c} /\left(b_{\text {idle, idle }}^{c}+L_{\text {data }} b_{\text {success }}^{c}+L_{\text {duration }} b_{\text {duration }}^{c}+L_{\text {ack }} b_{\text {ack }}^{c}+L_{\text {data }} b_{\text {failure }}^{c}+b_{\text {idle }}^{c}\right)
$$

\section{Results}

\subsection{Experimental Setup}

To measure the influence of traffic load on the adaptive CSMA-CA, we evaluate the performance of adaptive CSMA-CA in NS2 simulator with the following metrics. Throughput: The ratio of data transmission periods to all time that a transmission spends. Packet delivery rate (PDR): The ratio of packets successfully received to packets sent in MAC layer. This metric does not differentiate transmissions and retransmissions. Collision rate: The ratio of packets dropped by collisions to total packets sent in MAC layer. End-to-end MAC delay: The average transaction time of passing a packet from the source device to the destination device, including backoff time used in CSMA-CA and transmission over all successful end-to-end transmissions.

General parameters are set as follow. A star topology with a radius of 10 meters is used and there are six devices in it. Transmission range and carrier sense range can be set differently within a certain range $(15 \mathrm{~m}-30 \mathrm{~m})$ for testing the effects of hidden-terminals. PHY operates at an over air data rate of $250 \mathrm{kbps}$. The radio propagation model adopted is two-ray ground reflection. In the simulation, Poisson traffic is used and data frame length is assumed a fixed value of 90 bytes. Here BO and SO are both set 3 and the optional inactive part is not included in superframes. Traffic Load changes with arrival rate of the Poisson stream. Each independent simulation lasts 100 seconds and runs repeatedly with different random seeds.

\subsection{Performance Analysis in Scene without HT}

Figure 4 shows the effects of the traffic load on the throughput, packet delivery ratio, collision rate and average MAC delay including HT respectively.

In Figure 4(a), the throughput in Y-axis is normalized to the physical-layer transmission rate. This figures show that the adaptive CSMA-CA algorithm almost has the same performance as IEEE 802.15.4 standard when traffic load is light. However, it behaves much better as traffic load becomes heavier. Figure 4(b) gives out collision rate comparison. We find that the modified algorithm has an obvious improvement on collision avoidance. Fewer collisions also contribute to better throughput. But this curve is not quit smooth. This is because devices have a double size of the contention window via adaptiveBE parameter when most of data frames suffer collisions. Due to the gradient extension of the contention window, devices have obviously fewer chances of selecting the same backoff slot sending data in a quite short term. Thus, the adaptive CSMA-CA performance more effectively as traffic load becomes heavier. 
Figure 4(c) and Figure 4(d) respectively shows packet delivery ratio and MAC delay. Packet delivery ratio decreases heavier as the traffic load increases when system devices adopt the standard CSMA-CA. Even the adaptive algorithm increases contention window, it reduces the average delay efficiently under a heavier traffic load by avoiding plenty of retransmissions.

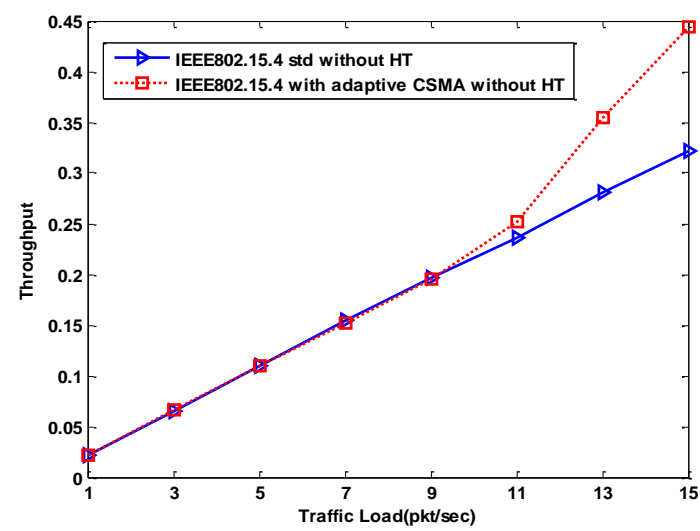

(a)

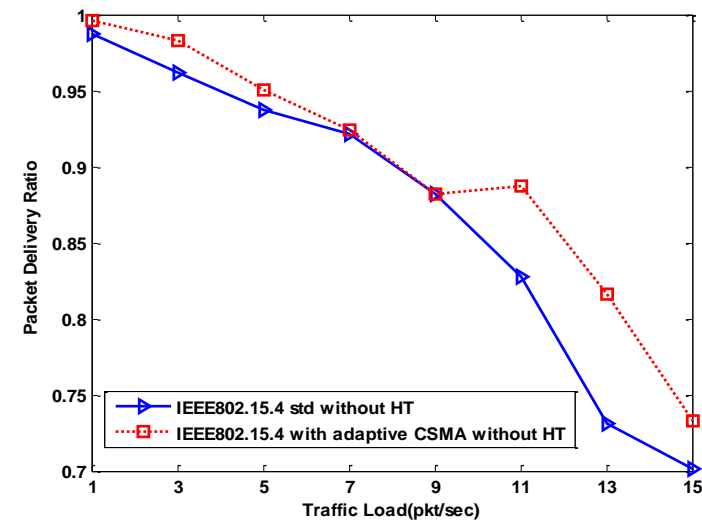

(c)

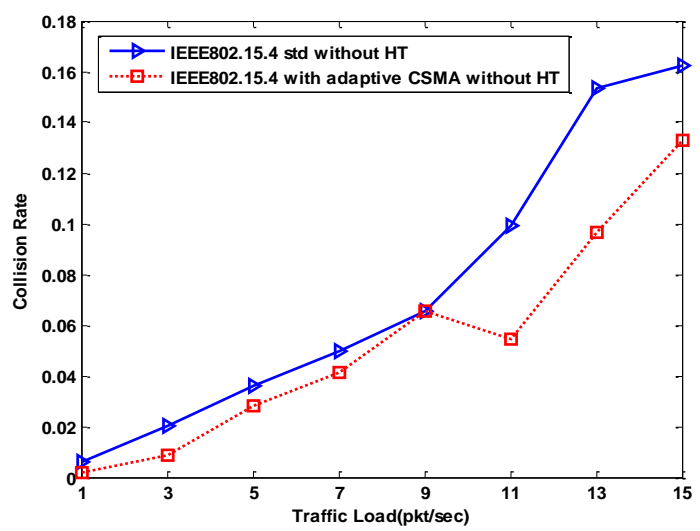

(b)

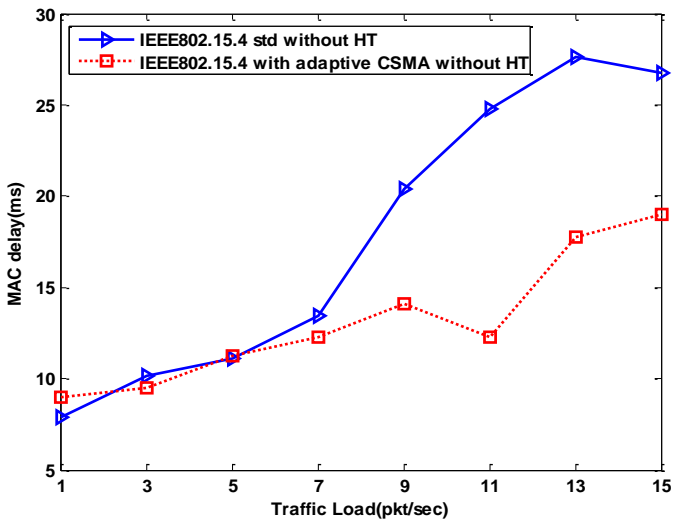

(d)

Figure 4. System Performance of Adaptive CSMA-CA: (a) Throughput, (b) Collision Rate, (c) Packet Delivery Rate, (d) MAC Delay

\subsection{Performance Analysis in Scene with HT}

The hidden terminal problem (HTP) is common in the wireless communication scenario using CSMA scheme in MAC layer. It is closely related to the Transmission Range (TX Range), Carrier Sensing Range (CS Range) and Interference Range (IF Range) of devices in wireless networks. The TX Range is the range within which a transmitted frame can be successfully received. The transmission ranges vary with different data rates, because different SNR requirements are designed for various data rates in PHY layer. The CS Range indicates the range within which devices can detect a transmission. The IF Range indicates the range within which devices in receiving mode can be interfered by a "hidden" transmitter.

Hidden-terminals refer to the nodes within the IF range of the intended destination and out of the CS of the source. As Figure 5 shows, device C and D are out of the CS range of device $\mathrm{B}$ but in the TX range of device A. The gray area indicates the hidden area. For C and D are hidden to B, they may access the channel at the same time and lead to a data collision. RTS/CTS mechanism is used in IEEE 802.11 to reduce HTP. The design of this mechanism is 
using RTS/CTS frames to tell neighbor nodes the channel will be occupied for a certain period. It effectively reduces collisions between hidden devices in 802.11. But the RTS/CTS mechanism is not adopted by IEEE 802.15.4 CSMA/CA.

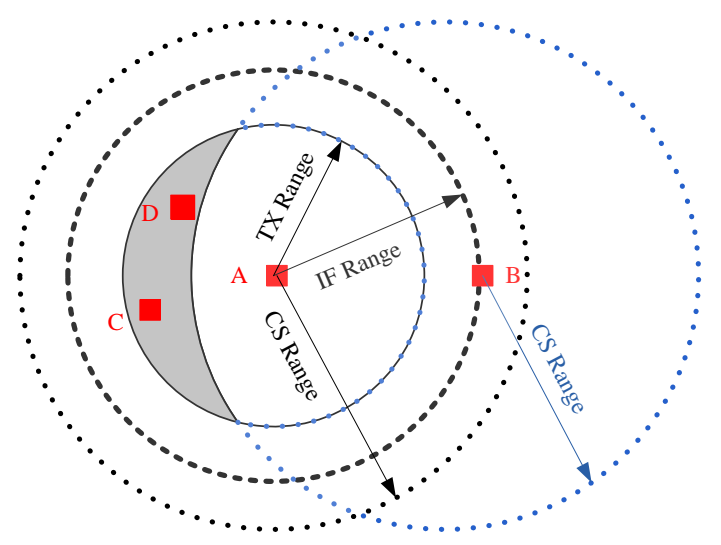

Figure 5. Hidden-terminals

In this part, we evaluate performance of the adaptive CSMA-CA respectively including hidden nodes. Figure 6 shows the effects of adaptive CSMA-CA algorithm on aspect of collision rate, packet delivery ratio. The range of traffic load in X-axis is set in a fairly small range for it performance declines too fast as traffic load varies.

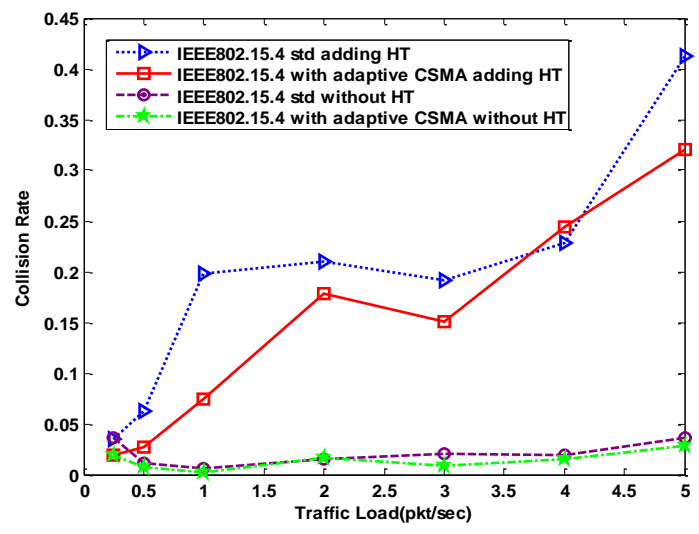

(a)

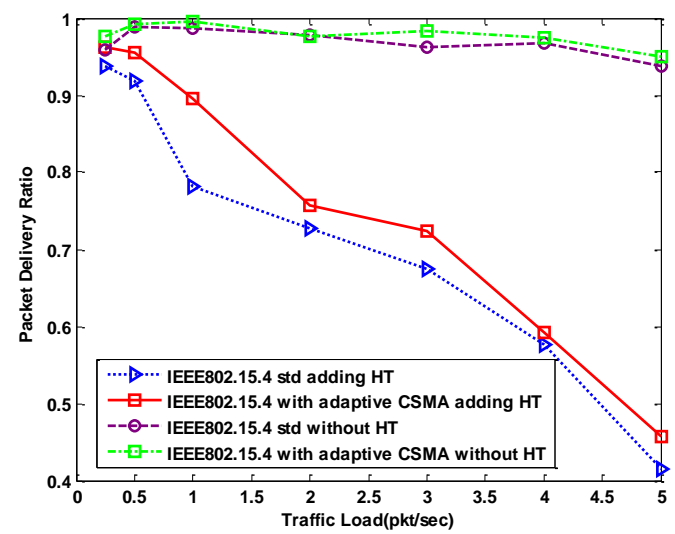

(b)

Figure 6. System Performance of Adaptive CSMA-CA in Scences Including HT: (a) Collision Rate, (b) Packet Delivery Rate

The adaptive algorithm has a little better performance compared with the standard in Figure 6. However we can find that the adaptive CSMA-CA algorithm cannot improve bad effects efficiently. Collisions in scenarios with HT can be attributed to two aspects. First, devices send data frame no matter whether the hidden nodes are transmitting data and CSMA-CA is ineffective for this collisions. Second, devices send data with other devices significantly in the sensing range when it detects the channel idle for the shortage CSMA-CA. According to comparison with the performance without HT in the same traffic load, we find that collisions caused by the first aspect are more serious than collisions by the second aspect. 
The adaptive CSMA-CA algorithm improves system performances by untargeted expending contention window to reduce collisions. But it fails to distinguish effects caused by traffic load and HT. When data exchanges between HTs are heavy, the adaptive algorithm cannot work effectively.

\section{Conclusion}

In this paper, we achieve dynamic-parameter CSMA-CA algorithm based traffic load estimation and analyzes its performance in direct transmission. It behaves well in scenarios excluding HT while improves performances little including HT. For a better property, feedback mechanism aiming at HT under different traffic load needs to be further studied.

\section{Acknowledgements}

This work in part was supported by Science and Technology Development Project of Shandong under Project No.2011GSF12007. Additional support is provided by a grant from Independent Innovation Foundation of Shandong University. The authors would express great gratitude to Dr. Zunhua Guo and Dr. Chengyou Wang for their help in the academic assistant. Special thanks also go to reviewers for their useful recommendations.

\section{References}

[1] G. Lu, B. Krishnamachari and C. S. Raghavendra, "Performance evaluation of the IEEE 802.15.4 MAC for low-rate low-power wireless networks", Proceedings of 23rd IEEE International Performance, Computing, and Communications Conference, (2004) April 15-17, Phoenix, United states.

[2] S. Wijetunge, U. Gunawardana and R. Liyanapathirana, "Impact of MAC parameters on the performance of IEEE 802.15.4 MAC protocol with ACK frame transmission", Proceedings of Australasian Telecommunication Networks and Applications Conference, (2011) October 31-November 3, Auckland, New Zealand.

[3] A. Faridi, M. R. Palattella, A. Lozano, M. Dohler, G. Boggia, L. A. Grieco and P. Camarda, "Comprehensive evaluation of the IEEE 802.15.4 MAC layer performance with retransmissions", IEEE Transactions on Vehicular Technology, vol. 59, no. 8, (2010), pp. 3917-3932.

[4] T. J. Lee, H. R. Lee and M. Y. Chung, "MAC throughput limit analysis of slotted CSMA/CA in IEEE 802.15.4 WPAN", IEEE Communications Letters, vol. 10, no. 7, (2006), pp. 561-563.

[5] J. Misic, S. Shafi and V. B. Misic, "Avoiding the bottlenecks in the MAC layer in 802.15.4 low rate WPAN", Proceedings of 11th International Conference on Parallel and Distributed Systems Workshops, (2005) July 20-22, Fukuoka, Japan.

[6] H. W. Tseng, A. C. Pang, J. Chen and C. F. Kuo, "An adaptive contention control strategy for IEEE 802.15.4-based wireless sensor networks", IEEE Transactions on Vehicular Technology, vol 58, no. 9, (2009), pp. 5164-5173.

[7] "IEEE Std 802.15.4-2003", IEEE Standard for Telecommunications and Information Exchange Between Systems - LAN/MWAN - Specific Requirments - Part 15: Wireless Medium Access Control (MAC) and Physical Layer (PHY) Specifications for Low Rate Wireless Personal Area Networks (WPANs), New York, United states, IEEE Standard, (2003).

\section{Authors}

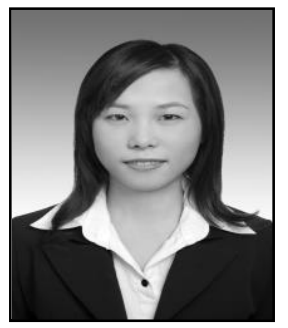

Lin Zhu, she received Bachelor degree from Shandong University, Weihai, China in 2011. Now she is studying at School of Mechanical, Information Engineering in Shandong University, Weihai, China. Her research interests focus on wireless sensor networks. 


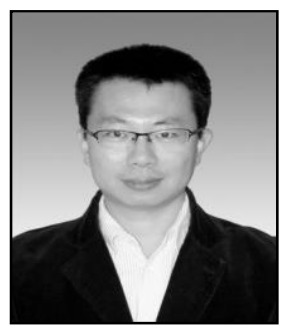

Guangming Li, he received Bachelor degree and Master degree from Shandong University in 1996 and 1999 respectively. He was engaged in research about fiber optic sensors in Texas A\&M University from 2000 to 2002. Then he enrolled at The University of Hong Kong and earned $\mathrm{Ph} . \mathrm{D}$. degree in 2006. Now he is working in Shandong University as an associate professor and has published over 30 papers in journals and international conferences about Optical networks and WSNs.

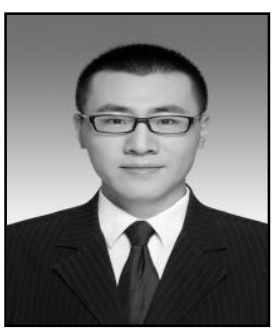

Wei Liu, he received Bachelor degree from Shandong University, Weihai, China in 2011. Now he is studying at School of Mechanical, Electrical and Information Engineering in Shandong University, Weihai, China. His research interests focus on wireless sensor networks. 